Noname manuscript No.

(will be inserted by the editor)

Valeria Ferrari · Leonardo Gualtieri

\title{
Quasi-Normal Modes and Gravitational Wave Astronomy
}

the date of receipt and acceptance should be inserted later

\begin{abstract}
We review the main results obtained in the literature on quasinormal modes of compact stars and black holes, in the light of recent exciting developments of gravitational wave detectors. Quasi-normal modes are a fundamental feature of the gravitational signal emitted by compact objects in many astrophysical processes; we will show that their eigenfrequencies encode interesting information on the nature and on the inner structure of the emitting source and we will discuss whether we are ready for a gravitational wave asteroseismology.
\end{abstract}

\section{Introduction}

In the past four years the sensitivity of the gravitational wave (GW) detectors LIGO and Virgo has been improved at a formidable rate [1. LIGO's noise curve has been lowered by about three orders of magnitude and now the detectors are operating at the design sensitivity. Similar progresses have been achieved by Virgo, although some effort remains to be done to reach the planned sensitivity at low frequencies $(\sim(10-40) \mathrm{Hz})$. In any event, these detectors are now in a position to take data good enough to start making science: a supernova exploding in the local group of nearby galaxies would not be missed, as well as the coalescence of compact bodies, neutron stars or black holes, with total mass smaller than about $100 M_{\odot}$, out to a distance of the orders of few Megaparsecs (these estimates are only indicative, since they are continuously updated as the detector sensitivities are improved). The detection of gravitational signals will allow to test the predictions of the theoretical work that has been done over the years to construct waveforms and energy spectra, and to extract distinctive features which could be traced back to the nature and to the structure of the source. An important piece

Dipartimento di Fisica "G. Marconi", Sapienza Università di Roma and INFN, Sezione Roma 1, P.le A. Moro 2, 00185 Roma, Italy 
of information is provided by the frequencies at which a compact object oscillates and emits gravitational waves, i.e the quasi-normal mode (QNM) frequencies. In this paper we shall discuss the pulsation properties of black holes and neutron stars, focussing in particular on the information they carry about the emitting source.

Accounts on quasi-normal modes of stars and black holes can also be found in [2] and [3].

\section{Do black holes oscillate?}

According to General Relativity, quasi-normal modes are the proper modes at which a black hole, or a star, oscillates when excited by a non radial perturbation. They are said quasi-normal, in contrast to the normal modes of Newtonian gravity, because they are damped by the emission of gravitational waves; as a consequence, the corresponding eigenfrequencies are complex. That a star can oscillate is, in some sense, obvious because a star is a ball of fluid 1]; however, when the idea that also black holes possess some proper modes of vibration was firstly proposed, it raised considerable surprise. Indeed, a black hole is not a material object, it is a singularity hidden by a horizon: how can it possibly oscillate? In order to understand how this bizarre behaviour was discovered, we need to go back to half a century ago and to the early theory of black hole perturbations.

\subsection{Schwarzschild perturbations are described by two wave equations}

In 1957 T. Regge and J.A. Wheeler [6 showed that the equations describing the perturbations of a Schwarzschild black hole can be separated if the perturbed metric tensor is expanded in tensorial spherical harmonics. They also showed that the relevant equations split into two decoupled sets belonging to different parity - $(-1)^{\ell}$ (even or polar) and $(-1)^{\ell+1}$ (odd or axial) and that, by a suitable choice of the gauge and by Fourier-expanding the perturbed functions, the equations for the radial part of the axial perturbations of a Schwarzschild black hole can be reduced to a single Schroedinger-like wave equation with a potential barrier, for a suitably defined function $Z_{\ell}^{-}$:

$$
\frac{d^{2} Z_{\ell}^{-}}{d r_{*}^{2}}+\left[\omega^{2}-V_{\ell}^{-}(r)\right] Z_{\ell}^{-}=0,
$$

where

$$
V_{\ell}^{-}(r)=\frac{1}{r^{3}}\left(1-\frac{2 M}{r}\right)[\ell(\ell+1) r-6 M], \quad r_{*}=r+2 M \log \left(\frac{r}{2 M}-1\right) .
$$

This equation is known as the Regge-Wheeler equation. A similar result was obtained later in 1970 by F. Zerilli [7, who showed that also the polar

\footnotetext{
1 The study of stellar oscillations started at the beginning of the past century, when Shapley 4] (1914) and Eddington [5] (1918) suggested that the variability observed in some stars is due to periodic pulsations.
} 
equations can be reduced to the wave equation (11) for a suitably defined function $Z_{\ell}^{+}$, and with a different potential barrier

$$
V_{\ell}^{+}(r)=\frac{2(r-2 M)}{r^{4}(n r+3 M)^{2}}\left[n^{2}(n+1) r^{3}+3 M n^{2} r^{2}+9 M^{2} n r+9 M^{3}\right],
$$

where $n=\frac{1}{2}(\ell-1)(\ell+2)$. If the perturbation is excited by a source, on the lefthand side there will be a forcing term obtained from the harmonic expansion of the stress-energy tensor of the exciting source. The wave equations for the axial and polar perturbations describe the way in which a non rotating black hole reacts to an external perturbation, and the gravitational signal emitted by the perturbed black hole can be calculated in terms of the two functions $Z_{\ell}^{-}$and $Z_{\ell}^{+}$as follows:

$h^{+}(t, r, \theta, \phi)=\frac{1}{2 \pi} \int \frac{e^{\mathrm{i} \omega\left(t-r_{*}\right)}}{r} \sum_{\ell m}\left[Z_{\ell m}^{+}(r, \omega) W^{\ell m}(\theta, \phi)-\frac{Z_{\ell m}^{-}(r, \omega)}{\mathrm{i} \omega} \frac{X^{\ell m}(\theta, \phi)}{\sin \theta}\right] d \omega$
$h^{\times}(t, r, \theta, \phi)=\frac{1}{2 \pi} \int \frac{e^{\mathrm{i} \omega\left(t-r_{*}\right)}}{r} \sum_{\ell m}\left[Z_{\ell m}^{+}(r, \omega) \frac{X^{l m}(\theta, \phi)}{\sin \theta}+\frac{Z_{\ell m}^{-}(r, \omega)}{\mathrm{i} \omega} W^{\ell m}(\theta, \phi)\right] d \omega$

where $h^{+}, h^{\times}$are the two polarizations of the gravitational wave in the transverse-traceless gauge (see [8], Chapter 35), and

$$
\begin{aligned}
W^{\ell m}(\theta, \phi) & =\left(\partial_{\theta}^{2}-\cot \theta \partial_{\theta}-\frac{1}{\sin ^{2} \theta} \partial_{\phi}^{2}\right) Y^{\ell m}(\theta, \phi) \\
X^{\ell m}(\theta, \phi) & =2\left(\partial_{\theta \phi}-\cot \theta \partial_{\phi}\right) Y^{\ell m}(\theta, \phi)
\end{aligned}
$$

where $Y^{\ell m}$ are scalar spherical harmonics. Note that, since we are considering perturbations of a spherically symmetric spacetime, $Z_{\ell m}^{ \pm}$coincides with $Z_{\ell}^{ \pm}$ for any value of $m$.

\subsection{Quasi-normal modes of a Schwarzschild black hole}

In 1970 Vishveshwara 9 pointed out that equation (11) for the functions $Z_{\ell}^{-}$and $Z_{\ell}^{+}$allows complex frequency solutions which satisfy the following boundary conditions

$$
\begin{aligned}
& Z_{\ell} \rightarrow e^{i \omega r_{*}}, \quad r_{*} \rightarrow-\infty, \\
& Z_{\ell} \rightarrow e^{-i \omega r_{*}}, \quad r_{*} \rightarrow+\infty ;
\end{aligned}
$$

the former represents a pure ingoing wave, since nothing can escape from a black hole horizon, the latter represents a pure outgoing wave at radial infinity and corresponds to the requirement that no radiation is incoming from infinity. This idea was confirmed by Press [10] who found, by integrating the wave equation numerically, that an arbitrary initial perturbation decays as a pure frequency mode. However, only in 1975 Chandrasekhar and Detweiler 
11] actually computed the discrete eigenfrequencies of these modes and clarified their nature. Quoting Chandrasekhar from its book The Mathematical Theory of Black Holes 12]:

".. we may expect on general grounds that any initial perturbation will, during its last stages, decay in a manner characteristic of the black hole and independently of the original cause. In other words, we may expect that during the very last stages, the black hole will emit gravitational waves with frequencies and rates of damping, characteristic of itself, in the manner of a bell sounding its last dying pure note. These considerations underlie the formulation of the concept of the quasi-normal modes of a black hole."

A Schwarzschild black hole is characterized by only one parameter, its mass $M$; consequently, the QNM frequencies depend only on $M$. In Table 1 we show the values of the complex characteristic frequencies of the first four QNMs of a Schwarzschild black hole, respectively for $\ell=2$ and $\ell=3$. In order to find the true pulsation frequency, $\nu$, and the damping time, $\tau$,

Table 1 The lowest QNM frequencies of a Schwarzschild black hole for $\ell=2$ and $\ell=3$. They are the same both for the polar and for the axial perturbations, i.e. the two potential barriers (2) and (3) are isospectral.

\begin{tabular}{|c|c|c|c|}
\hline & $M \omega_{0}+i M \omega_{i}$ & & $M \omega_{0}+i M \omega_{i}$ \\
\hline$\ell=2$ & $0.3737+\mathrm{i} 0.0890$ & $\ell=3$ & $0.5994+\mathrm{i} 0.0927$ \\
\hline & $0.3467+\mathrm{i} 0.2739$ & & $0.5826+\mathrm{i} 0.2813$ \\
\hline & $0.3011+\mathrm{i} 0.4783$ & & $0.5517+\mathrm{i} 0.4791$ \\
\hline & $0.2515+\mathrm{i} 0.7051$ & & $0.5120+\mathrm{i} 0.6903$ \\
\hline
\end{tabular}

from the values given in Table 1, we proceed as follows. Let us assume that the black hole mass is $M=n M_{\odot}, \quad\left(M_{\odot}=1.48 \cdot 10^{5} \mathrm{~cm}\right)$; converting to physical unities we find

$\nu=\frac{c}{2 \pi n \cdot M_{\odot}\left(M \omega_{0}\right)}=\frac{32.26}{n}\left(M \omega_{0}\right) \mathrm{kHz}, \quad \tau=\frac{n M_{\odot}}{\left(M \omega_{i}\right) c}=\frac{n \cdot 0.4937 \cdot 10^{-5}}{\left(M \omega_{i}\right)} \mathrm{s}$.

Using these expressions we can check whether a gravitational signal emitted by an oscillating black hole falls within the bandwidth of the ground based interferometers Virgo/LIGO or within that of the space based interferometer LISA. Virgo/LIGO bandwidth extends over a range of frequencies which goes from about $10-40 \mathrm{~Hz}$, up to few $\mathrm{kHz}$. Thus, these detectors will be able to detect the signal emitted by an oscillating black hole (if it is sufficiently strong) with mass ranging within

$$
10 M_{\odot} \lesssim M \lesssim 10^{3} M_{\odot}
$$

corresponding to the frequency range $\nu \in[12 \mathrm{~Hz}, 1.2 \mathrm{kHz}]$; LISA will be sensitive to the frequency region $\nu \in\left[10^{-4}, 10^{-1}\right] \mathrm{Hz}$, and will see oscillating black holes with mass

$$
1.2 \cdot 10^{5} M_{\odot} \lesssim M \lesssim 1.2 \cdot 10^{8} M_{\odot} .
$$


For instance, LISA will be able to detect signals emitted by the oscillations of the massive black hole at the center of our Galaxy SGR A*, the mass of which is $M=(3.7 \pm 0.2) \cdot 10^{6} M_{\odot}[13$.

While the frequencies of the lowest modes are rather easy to compute, great care must be used to determine the entire spectrum. Many different methods have been used to this purpose. For instance, a WKB approximation and a higher order WKB approach have been used to find the lowest [14] and the higher mode frequencies [15], respectively. In addition, new approaches have been developed to study the QNM spectrum, as the continued fraction method (developed for Kerr black holes in [16]) and the phase-integral method [17]. Using these approaches, it has been found [18] that, for any value of the harmonic index $\ell$, as the order $n$ of the mode increases, the real part of a mode frequency approaches a non zero limiting value. Furthermore, an analytical expression has been found for the imaginary part of the frequency, valid in the limit $n \rightarrow \infty$. We also mention that an exact, analytical solution of the Regge-Wheeler equation has recently been found in terms of the Heun functions. QNM eigenfrequencies can be computed in terms of this solution, by solving numerically a boundary value problem [19].

\subsection{The quasi-normal modes of a Kerr black hole}

After 1975 the study of black hole perturbations follows along two principal avenues. One studies directly the perturbations of the metric tensor via Einstein's equations linearized about a given background. The other studies the perturbations of Weyl's and Ricci's scalars using the Newman-Penrose formalism. Using this latter approach in 1972 Teukolsky 20 was able to decouple and separate the equations governing the perturbations of a Kerr black hole, and to reduce them to a single master equation for the radial part of the perturbation $R_{\ell m}$ :

$$
\left\{\begin{array}{l}
\Delta R_{\ell m, r r}+2(s+1)(r-M) R_{\ell m, r}+V(r) R_{\ell m}=0 \\
V_{\ell m}(r, \omega)=\frac{1}{\Delta}\left[\left(r^{2}+a^{2}\right)^{2} \omega^{2}-4 a M r m \omega+a^{2} m^{2}+2 i s(a m(r-M)\right. \\
\left.\left.-M \omega\left(r^{2}-a^{2}\right)\right)\right] r+\left[2 i s \omega r-a^{2} \omega^{2}-A_{\ell m}\right] \\
\Delta=r^{2}-2 M r+a^{2}
\end{array}\right.
$$

The angular part, $S_{\ell m}$, satisfies the equations of the oblate spheroidal harmonics

$$
\left\{\begin{array}{l}
{\left[\left(1-u^{2}\right) S_{\ell m, u}\right]_{, u}+\left[a^{2} \omega^{2} u^{2}-2 a m \omega s u+s+A_{\ell m}-\frac{(m+s u)^{2}}{1-u^{2}}\right] S_{\ell m}=0} \\
u=\cos \theta
\end{array}\right.
$$

and the complete wavefunction is

$$
\psi_{s}(t, r, \theta, \varphi)=\frac{1}{2 \pi} \int e^{\mathrm{i} \omega t} \sum_{\ell=|s|}^{\infty} \sum_{m=-\ell}^{\ell} e^{i m \varphi} S_{\ell m}(u) R_{\ell m}(r) d \omega
$$


In these equations $s$ is the spin-weight parameter $s=0, \pm 1, \pm 2$, for scalar, electromagnetic and gravitational perturbations, respectively 2 , and $A_{\ell m}$ is a separation constant.

It is interesting to note that, unlike the potential barrier of a Schwarzschild black hole, which is real and independent of the harmonic index $m$ and of the frequency, the potential barrier of a Kerr black hole is complex, and it depends on $m$ and on the frequency $\omega$.

An interesting phenomenon occurs when electromagnetic or gravitational waves are scattered by Kerr's potential barrier $V_{\ell m}(r, \omega)$; if the incident wave has a frequency in the range

$$
0<\omega<\omega_{c} \quad \text { where } \quad \omega_{c}=\frac{a m}{2 M r_{+}}, \quad m>0
$$

the reflection coefficient associated to $V(r)$ exceeds unity [21,22. This phenomenon is called superradiance, and it is the analogue, in the domain of wave propagation, of Penrose's process in the domain of particle creation.

The quasi-normal frequencies of a Kerr black hole have been computed by Detweiler [23], and subsequently by Leaver [16], Seidel \& Iyer [24], Kokkotas 25] and Onozawa [26]. Since rotation removes the degeneracy presented by Schwarzschild's modes, there is a set of eigenmodes for any assigned value of the harmonic indexes $\ell$ and $m$. The calculations show that when the black hole angular momentum $a$ increases, the real part of the complex eigenfrequencies is bounded, but the imaginary part is not. Moreover, when a Kerr black hole becomes "extreme", i.e. when $a \rightarrow M$, highly damped modefrequencies converge to the purely real value of the critical frequency below which superradiant scattering occurs, $\omega_{c}=\frac{m}{2 M}$. In this context, an interesting result was obtained by Detweiler in 1977 [23. He found that when $a \rightarrow M$, the imaginary part of the mode-frequencies tends to zero. If excited, these modes would set the black hole into an oscillation that would never decay, suggesting that extreme Kerr black holes are "marginally unstable". It was subsequently shown by B. Mashoon and one of the authors that when $a \rightarrow M$ the amplitude of the "unstable" modes tends to zero, and consequently quasi-normal modes belonging to real frequency cannot exist in the ordinary regime [27].

As for a Schwarzschild black hole, in the $n \rightarrow \infty$ limit the imaginary part of the mode frequencies has an analytical expression which has been determined in [28].

\subsection{Excitation of black hole quasi-normal modes}

Black holes quasi-normal modes are excited in many astrophysical processes, and are a fundamental feature of the gravitational signal. Therefore they are of utmost importance for the data analysis of gravitational wave experiments. The first simulation of black hole oscillations excited by an external

\footnotetext{
${ }^{2}+$ and - indicate the ingoing and outgoing radiative part of the considered field. For example $s=+2$ corresponds to $\psi_{2}=\Psi_{0}$, and $s=-2$ to $\psi_{-2}=\Psi_{4}$, where $\Psi_{0}$ and $\Psi_{4}$ are the Weyl scalars.
} 
source dates back to 1971: in 29] a Schwarzschild black hole was perturbed by a radially infalling point-like body, with a mass much smaller than the black hole mass. The energy spectrum of the emitted signal was computed by solving the Zerilli equation with a source describing the infalling particle. The waveform was explicitly computed in [30, and it was shown that, after a transient, the signal exhibits a ringing tail, which can be fitted by a combination of quasi-normal modes. In Figure 1, we show the gravitational wave amplitude $r \cdot h\left(t-r_{*}\right)$ emitted in the considered process, and the analytical fit with the first two $\ell=2$ quasi-normal modes belonging to the frequencies $M \omega_{1}=0.37+\mathrm{i} 0.09$ and $M \omega_{2}=0.35+\mathrm{i} 0.27$. The fit becomes more accurate if higher order modes are taken into account, but the main contribution is due to $\omega_{1}$ and $\omega_{2}$.

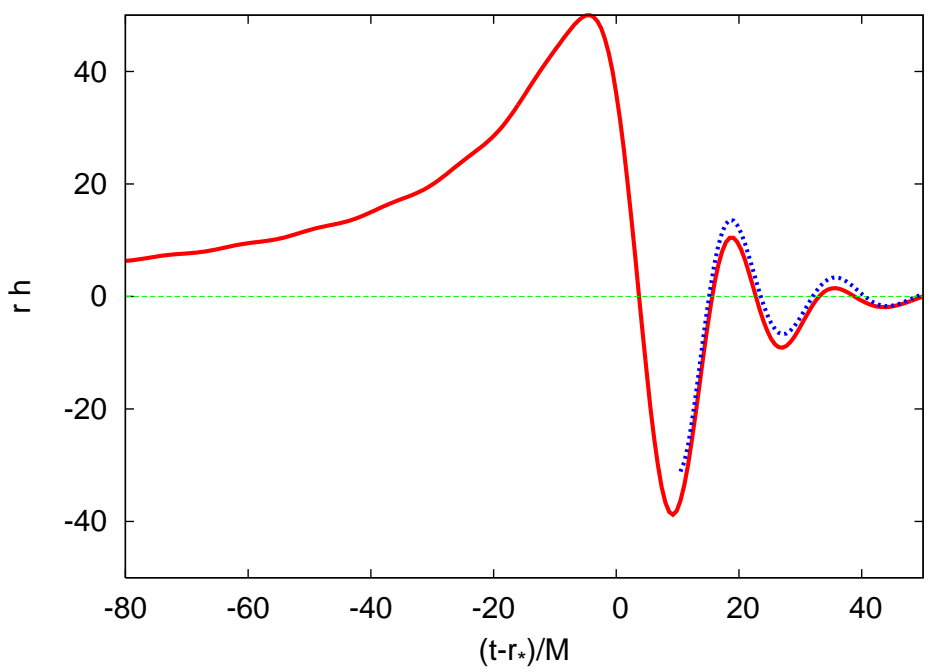

Fig. 1 The gravitational signal emitted when a Schwarzschild black hole is perturbed by a radially infalling particle (solid line); the analytical fit (dashed line) has been obtained using a linear combination of the first two $\ell=2$ black hole quasi-normal modes.

The perturbation induced on a Schwarzschild black hole by extended sources made up of pressureless matter was studied in [31. It was shown that in this case modes are also excited, but to a smaller extent with respect to the pointlike source, due to interference effects (see also [32,33]). In the case of matter orbiting around a black hole, the excitation of the QNMs is in general even smaller. Indeed, modes are significantly excited only if matter reaches $r \lesssim 4 M$, i.e., if it crosses the potential barrier $V_{\ell}^{ \pm}(r)$; however, this is not possible if matter moves on a stable orbit, for which $r \geq 6 M[34$.

Although these studies refer to very idealised situations, they have been very useful because they showed that quasi-normal modes can be excited, and because they provided a first understanding of the mechanisms underlying 
the mode excitation. However, astrophysical phenomena are much more complicate, and only recently major advances in numerical techniques allowed the modelling of more realistic processes involving black holes.

Black hole coalescence is probably the most violent process occurring in the universe (after the big bang), and it is expected to be the most powerful source of gravitational waves to be detected by interferometric detectors Virgo and LIGO.

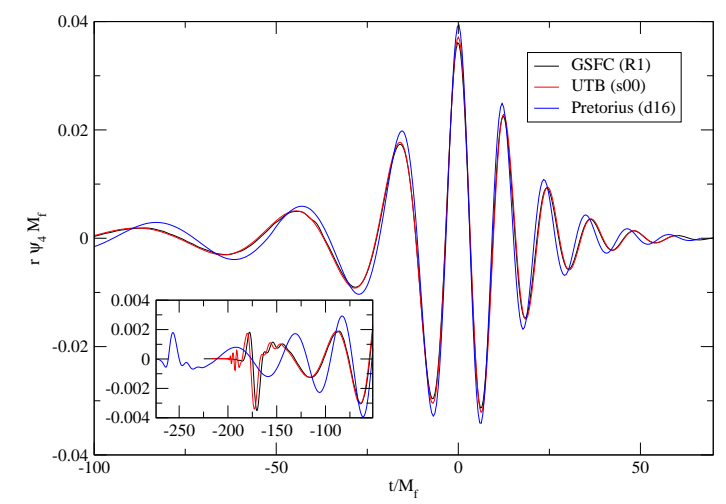

Fig. 2 Gravitational radiation waveform emitted by two coalescing black holes (courtesy of the authors).

Such phenomena are very difficult to simulate numerically, because the topology of the spacetime changes during the process. After nearly 15 years of efforts, this problem has been solved very recently by three groups [35, who found, independently, the same results [36. In their simulations, two Schwarzschild black holes coalesce, merge and produce a single Kerr black hole. The emitted gravitational signal computed in [36] is shown in Figure2, after a short merger phase the waveform is clearly dominated by the quasinormal mode oscillations. This important feature is also exhibited by the signal emitted in the coalescence of rotating black holes 37 .

Quasi-normal modes excitation has also been shown to give a strong contribution to the signal emitted after the core collapse of a rotating neutron star to a Kerr black hole 38. Moreover, in 39] the gravitational emission of a black hole perturbed by a thick, oscillating accretion disk has been studied; in a subsequent paper [40] it has been shown that if the disk is extremely dense, black hole oscillations can be excited, even though, due to the symmetry of the source, only by a small amount. All numerical simulations of astrophysical processes in which QNMs are excited show that the leading contribution belong to the lowest frequency, $\ell=2$ mode.

To conclude this Section, we should at least mention that there are also theoretical studies [41, [42] on the "excitability" of the quasi-normal modes. The main result of these studies is that a measure of the relative QNM excitation, independent of the particular astrophysical process, is encoded 
in the poles of the Green's functions associated to the Zerilli and ReggeWheeler equations. This information, combined with the knowledge of the exciting source, allows to determine the quasi-normal mode content of the gravitational signal.

\subsection{Other issues on quasi-normal modes}

\subsubsection{Completeness}

The quasi-normal modes of a Schwarzschild black hole do not form a complete basis for black hole perturbations. As shown by Leaver [41, this is due to a branch cut in the Green's functions associated to the Zerilli and ReggeWheeler equations. Consequently, a general perturbation cannot be written as a combination of quasi-normal modes. In particular, it exhibit a power-law tail $\sim t^{-2 \ell-2}$ 43.

\subsubsection{Stability}

The stability of the Schwarzschild spacetime has been proved by Vishveshwara [4] and Wald [45]. Vishveshvara showed that the imaginary part, $\omega_{i}$, of the QNM frequency is always positive, and Wald proved that since $\omega_{i}$ is always positive, all perturbations remain bounded.

The stability of Kerr black holes is still an open issue. The main problem arises because of the existence of the ergoregion, where a perturbation can grow indefinitely even though the energy remains finite. However, there are indications that also Kerr black holes are stable [46].

\subsubsection{Applications in string theory and in loop quantum gravity}

In recent years, it has been suggested that black hole QNM's may play a role in string theory and loop quantum gravity. In 2000 Horowitz and Hubeny [4] proposed that the study of the black hole QNM's in anti-de Sitter spacetime could be useful to determine some properties of conformal field theories. Their conjecture is deeply rooted in string theory and in the so-called "AdS-CFT correspondence". Stimulated by this work, many authors computed the QNM eigenfrequencies in anti-de Sitter spacetime [48] (see also [49]). It is worth reminding that anti-de Sitter solution of Einstein's equations describes a universe with a negative cosmological constant; therefore these black holes should not be considered as astrophysical objects.

In 2003 Dreyer and Motl [50] suggested that, in the asymptotic limit $n \rightarrow \infty$, black hole quasi-normal modes would allow to fix the value of the "Immirzi parameter", which is a key parameter in loop quantum gravity. Following this proposal, studies of the asymptotic limit of QNM [18, 28] have further been developed 51 .

More generally, inspired by these consideration in the contexts of string theory and loop quantum gravity, in recent years many authors have computed the eigenfrequencies of black hole quasi-normal modes in various back- 
ground spacetimes, both in four dimensions and for higher dimensional spacetimes 52 .

\section{Stellar pulsations}

Stellar pulsations are a very well known phenomenon in astronomy, since they underlay a variety of astrophysical processes. For instance, they are observed in the Sun, and a branch of solar sciences, named helioseismology, uses the information encoded in the pulsation frequencies to investigate the internal structure of our star and the physical processes that occur in the interior. Non radial pulsations are associated to gravitational wave emission and, as we shall see, the mode frequencies carry interesting information on the inner structure of the emitting sources. Thus, if in the future GW-detectors will be able to catch the gravitational signals emitted by pulsating stars, a new branch of astrophysics will develop, the gravitational wave asteroseismology. This will allow us to investigate the interior of neutron stars, where densities and pressures are so extreme that they are unreachable by high energy experiments on Earth. But before discussing how the equation of state (EOS) of matter affects quasi-normal mode frequencies, let us preliminarily show the equations we need to solve to determine these frequencies. We shall discuss only pulsations of a non rotating star, i.e. of stars which are described by static, spherically symmetric solutions of Einstein's equations. The rotating case is much more complicate, and an exhaustive description of the problems that arise when one is looking for the quasi-normal mode frequencies is beyond the scope of this paper (see, for instance, [53] and references therein).

\subsection{Stellar perturbations of a non rotating star}

The equations governing the adiabatic perturbations of a spherical star in general relativity have been derived within different approaches by many authors [54-60]. Here we shall show, as an example, the basic equations of the theory of stellar perturbations as developed in [55] (see also [56] for a complete account of the theory). We start with the metric appropriate to describe a static, spherical background

$$
d s^{2}=e^{2 \nu}(d t)^{2}-e^{2 \psi} d \varphi-e^{2 \mu_{2}}(d r)^{2}-e^{2 \mu_{3}}(d \theta)^{2} .
$$

where $e^{2 \psi}=r^{2}, e^{2 \mu_{3}}=r^{2} \sin ^{2} \theta$, and $\nu(r)$ and $\mu_{2}(r)$ have to be found by solving the TOV equations of stellar structure (see for instance [8]), for an assigned equation of state. Matter in the star is assumed to be a perfect fluid, with stress-energy tensor

$$
T^{\mu \nu}=(\epsilon+p) u^{\mu} u^{\nu}-p g^{\mu \nu},
$$

where $\epsilon(r)$ and $p(r)$ are the fluid energy-density and the pressure, and $u^{\mu}$ are the components of the four-velocity of a generic fluid element. Axisymmetric perturbations of the spacetime (11) can be described by the line-element

$$
d s^{2}=e^{2 \nu}(d t)^{2}-e^{2 \psi}\left(d \varphi-q_{2} d r^{2}-q_{3} d \theta-\omega d t\right)^{2}-e^{2 \mu_{2}}(d r)^{2}-e^{2 \mu_{3}}(d \theta)^{2} .
$$


In eq. (12) there are seven unknown functions, i.e. one more than needed, but the extra degree of freedom disappears when the boundary conditions of the problem are fixed. As a consequence of a generic perturbation, the metric functions, $\left(\nu, \psi, \mu_{2}, \mu_{3}, \omega, q_{2}, q_{3}\right)$, and the fluid variables, $\left(\epsilon, p, u^{\alpha}\right)$, change by a small amount with respect to their unperturbed values, which we assume to be known; for instance $\nu \longrightarrow \nu+\delta \nu, \epsilon \longrightarrow \epsilon+\delta \epsilon$, and similarly for the remaining variables. At the same time, each element of fluid undergoes an infinitesimal displacement from its equilibrium position, which is described by the lagrangian displacement $\boldsymbol{\xi}$. All perturbed quantities are functions of $t, r$ and $\theta$. If we now write Einstein's equations supplemented by the hydrodynamical equations and the conservation of baryon number, expand all tensors in tensorial spherical harmonics and Fourier-expand the time dependent quantities, we find that, as for black holes, the perturbed equations decouple into two sets, the polar and the axial, with a major difference: the polar equations couple the thermodynamical variables to the metric variables. Conversely the axial perturbations do not induce fluid motion except for a stationary rotation; however, we shall see that the fluid plays a role, because it shapes the potential barrier associated to the spacetime curvature.

\subsubsection{The equations for the polar perturbations}

The explicit expressions of the functions that describe the polar perturbations, expanded in harmonics and Fourier-expanded are

$$
\begin{array}{cl}
\delta \nu=N_{\ell}(r) P_{\ell}(\cos \theta) e^{i \omega t} & \delta \mu_{2}=L_{\ell}(r) P_{\ell}(\cos \theta) e^{i \omega t} \\
\delta \mu_{3}=\left[T_{\ell}(r) P_{\ell}+V_{\ell}(r) P_{\ell, \theta, \theta}\right] e^{i \omega t} & \delta \psi=\left[T_{\ell}(r) P_{\ell}+V_{\ell}(r) P_{\ell, \theta} \cot \theta\right] e^{i \omega t}, \\
\delta p=\Pi_{\ell}(r) P_{\ell}(\cos \theta) e^{i \omega t} & 2(\epsilon+p) e^{\nu+\mu_{2}} \xi_{r}(r, \theta) e^{i \omega t}=U_{\ell}(r) P_{\ell} e^{i \omega t} \\
\delta \epsilon=E_{\ell}(r) P_{\ell}(\cos \theta) e^{i \omega t} & 2(\epsilon+p) e^{\nu+\mu_{3}} \xi_{\theta}(r, \theta) e^{i \omega t}=W_{\ell}(r) P_{\ell, \theta} e^{i \omega t},
\end{array}
$$

where $P_{\ell}(\cos \theta)$ are Legendre's polynomials and $\omega$ is the frequency. After separating the variables the relevant Einstein's equations become

$$
\left\{\begin{array}{l}
X_{\ell, r, r}+\left(\frac{2}{r}+\nu_{, r}-\mu_{2, r}\right) X_{\ell, r}+\frac{n}{r^{2}} e^{2 \mu_{2}}\left(N_{\ell}+L_{\ell}\right)+\omega^{2} e^{2\left(\mu_{2}-\nu\right)} X_{\ell}=0 \\
\left(r^{2} G_{\ell}\right)_{, r}=n \nu_{, r}\left(N_{\ell}-L_{\ell}\right)+\frac{n}{r}\left(e^{2 \mu_{2}}-1\right)\left(N_{\ell}+L_{\ell}\right)+r\left(\nu_{, r}-\mu_{2, r}\right) X_{\ell, r}+\omega^{2} e^{2\left(\mu_{2}-\nu\right)} r X_{\ell} \\
-\nu_{, r} N_{\ell, r}=-G_{\ell}+\nu_{, r}\left[X_{\ell, r}+\nu_{, r}\left(N_{\ell}-L_{\ell}\right)\right]+\frac{1}{r^{2}}\left(e^{2 \mu_{2}}-1\right)\left(N_{\ell}-r X_{\ell, r}-r^{2} G_{\ell}\right) \\
-e^{2 \mu_{2}}(\epsilon+p) N_{\ell}+\frac{1}{2} \omega^{2} e^{2\left(\mu_{2}-\nu\right)}\left\{N_{\ell}+L_{\ell}+\frac{r^{2}}{n} G_{\ell}+\frac{1}{n}\left[r X_{\ell, r}+(2 n+1) X_{\ell}\right]\right\} \\
L_{\ell, r}(1-D)+L_{\ell}\left[\left(\frac{2}{r}-\nu_{, r}\right)-\left(\frac{1}{r}+\nu_{, r}\right) D\right]+X_{\ell, r}+X_{\ell}\left(\frac{1}{r}-\nu_{, r}\right)+D N_{\ell, r}+ \\
+N_{\ell}\left(D \nu_{, r}-\frac{D}{r}-F\right)+\left(\frac{1}{r}+E \nu_{, r}\right)\left[N_{\ell}-L_{\ell}+\frac{r^{2}}{n} G_{\ell}+\frac{1}{n}\left(r X_{\ell, r}+X_{\ell}\right)\right]=0
\end{array}\right.
$$

where

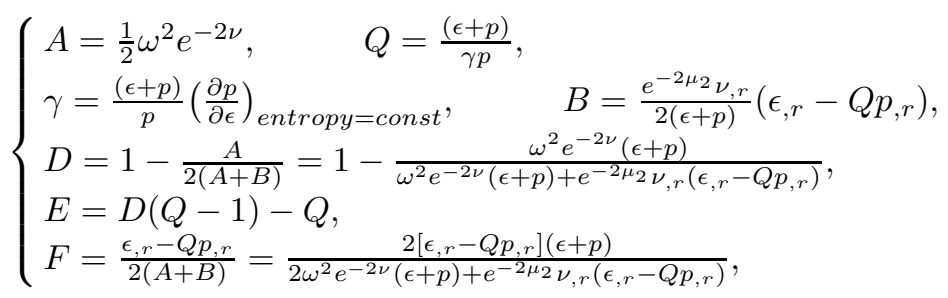


and $V_{\ell}$ and $T_{\ell}$ have been replaced by $X_{\ell}$ and $G_{\ell}$ defined as

$$
\left\{\begin{array}{l}
X_{\ell}=n V_{\ell} \\
G_{\ell}=\nu_{, r}\left[\frac{n+1}{n} X_{\ell}-T_{\ell}\right]_{, r}+\frac{1}{r^{2}}\left(e^{2 \mu_{2}}-1\right)\left[n\left(N_{\ell}+T_{\ell}\right)+N_{\ell}\right] \\
+\frac{\nu_{, r}}{r}\left(N_{\ell}+L_{\ell}\right)-e^{2 \mu_{2}}(\epsilon+p) N_{\ell}+\frac{1}{2} \omega^{2} e^{2\left(\mu_{2}-\nu\right)}\left[L_{\ell}-T_{\ell}+\frac{2 n+1}{n} X_{\ell}\right] .
\end{array}\right.
$$

Equations (14) are valid in general, also for non-barotropic equations of state.

It should be noted that although eqs. (14) describe stellar perturbations inside the star, they are written for the variables $(X, G, N, L)$ which are metric perturbations. However, the motion of the fluid is excited by the perturbation and it can be shown that, once equations (14) have been solved, the fluid variables, $(\Pi, E, U, W)$, can be obtained in terms of the metric functions using the following equations

$$
\begin{aligned}
& W_{\ell}=T_{\ell}-V_{\ell}+L_{\ell}, \\
& \Pi_{\ell}=-\frac{1}{2} \omega^{2} e^{-2 \nu} W_{\ell}-(\epsilon+p) N_{\ell}, \quad E_{\ell}=Q \Pi_{\ell}+\frac{e^{-2 \mu_{2}}}{2(\epsilon+p)}\left(\epsilon_{, r}-Q p_{, r}\right) U_{\ell}, \\
& \left.\left.U_{\ell}=\frac{\left[\left(\omega^{2} e^{-2 \nu} W_{\ell}\right)_{, r}+(Q+1) \nu_{, r}\left(\omega^{2} e^{-2 \nu} W_{\ell}\right)+2\left(\epsilon_{, r}-Q p_{, r}\right) N_{\ell}\right](\epsilon+p)}{\left[\omega^{2} e^{-2 \nu}(\epsilon+p)+e^{-2 \mu_{2}} \nu_{, r}(\epsilon, r\right.}-Q p_{, r}\right)\right]
\end{aligned}
$$

This fact is remarkable: it shows that all information on the dynamical behaviour of a star is encoded in the gravitational field. Thus, if one is interested exclusively in the study of the emitted gravitational radiation, one can solve the system (14) disregarding the fluid variables 3 .

Equations (14) have to be integrated for assigned values of the frequency from $r=0$, where all functions must be regular, up to the stellar surface. There, the spacetime becomes vacuum and spherically symmetric, and the perturbed metric functions and their first derivatives have to be matched continuously with the Zerilli function that describes the polar perturbations of a Schwarzschild spacetime; its expression in terms of the metric functions is

$$
Z_{\ell}^{+}(r)=\frac{r}{n r+3 M}\left(3 M X_{\ell}(r) / n-r L_{\ell}(r)\right)
$$

(for a detailed discussion of the boundary conditions see refs. [55] and [56]).

\subsubsection{A Schroedinger equation for the axial perturbations}

The equations for the axial perturbations are much simpler than the polar ones. Their radial behaviour is completely described by a function $Z_{\ell}^{-}(r)$, which satisfies the following Schroedinger-like equation

$$
\frac{d^{2} Z_{\ell}^{-}}{d r_{*}^{2}}+\left[\omega^{2}-V_{\ell}^{-}(r)\right] Z_{\ell}^{-}=0
$$

where $r_{*}=\int_{0}^{r} e^{-\nu+\mu_{2}} d r$ ( $\nu$ and $\mu_{2}$ are unperturbed metric functions), and

$$
V_{\ell}^{-}(r)=\frac{e^{2 \nu(r)}}{r^{3}}\left\{\ell(\ell+1) r+r^{3}[\epsilon(r)-p(r)]-6 m(r)\right\} .
$$

\footnotetext{
3 After these equations were derived, R.Ipser and R.H.Price showed that they can be reduced to a fourth-order system 61.
} 
The function $Z_{\ell}^{-}(r)$ is a combination of the axial, metric perturbations

$$
e^{3 \psi+\nu-\mu_{2}-\mu_{3}}\left(\delta q_{2, \theta}-\delta q_{3, r}\right)=r Z_{\ell}^{-}(r) C_{\ell+2}^{-\frac{3}{2}}(\theta),
$$

and $C_{\ell+2}^{-\frac{3}{2}}(\theta)$ are Gegenbauer's polynomials [55].

Outside the star $\epsilon$ and $p$ vanish and $V_{\ell}^{-}(r)$ reduces to the Regge-Wheeler potential barrier (2). From these equations we see that unlike the polar perturbations, the axial perturbations, which do not have a Newtonian counterpart, are not coupled to fluid motion. In addition, the shape of the potential (19) depends on $\epsilon(r)$ and $p(r)$, i.e. on the radial profile of the energy-density and of the pressure inside the star, in the equilibrium configuration.

\subsection{Quasi-normal modes of stars}

The quasi-normal modes are solutions of the axial and polar equations that satisfy the following boundary conditions. As for black holes, at radial infinity the solution must behave as a pure outgoing wave

$$
Z_{\ell}^{ \pm} \rightarrow e^{-i \omega t}, \quad r_{*} \rightarrow+\infty
$$

In addition, all perturbed functions must be regular at $r=0$ and have to match continuously the exterior perturbation on the stellar surface. For the axial perturbations the matching condition is automatically satisfied, because eq. (18) reduces to the Regge-Wheeler equation for $r \geq R$, where $R$ is the stellar radius.

Stars possess many different classes of modes. The axial quasi-normal modes are pure spacetime modes and do not exist in Newtonian gravity. They are named $w$-modes and are highly damped, i.e. the imaginary part of the frequency is comparable to the real part 62 and consequently the damping times are small. If the star is extremely compact, the potential (19) inside the star becomes a well, while in the exterior it remains a barrier. If the well is deep enough, it allows for the existence of one or more slowly damped quasi-normal modes, or $s$-modes; they are also said trapped modes because, due to the slow damping, they are effectively trapped by the potential barrier, and no much radiation can leak out of the star when these modes are excited 63 .

It is interesting to compare the eigenfrequencies of the axial modes of stars and black holes, since they are both pure spacetime modes. As an example, in table 2 we show the frequencies and the damping times of the first four $\ell=2$, axial modes for a homogeneous star with mass $M=1.35 M_{\odot}$ and increasing compactness, and for a non rotating black hole with the same mass. It should be mentioned that the $w$-mode frequencies depend upon the equation of state of matter in the inner core of the star and, as shown in 64, if detected they would allow to discriminate between the models underlying different equations of state. Until very recently, the common belief was that $w$-modes are unlikely to be excited in astrophysical processes. However in 2005 it has been shown that, in the collapse of a neutron star to a black hole, $w$-modes are excited soon before the black hole forms. Thus, the gravitational 
Table 2 The characteristic frequencies and damping times of the axial quasinormal modes of a homogeneous star of mass $M=1.35 M_{\odot}$. The data are tabulated for increasing values of the stellar compactness $\frac{M}{R}$; they are compared to those of a non rotating black hole with the same mass. We tabulate the first four values of the frequency (in $\mathrm{kHz}$ ) and of the damping time (in s) for $\ell=2 . \nu^{s}$ and $\tau^{s}$ refer to the trapped modes associated to the potential well inside the star (see text), $\nu^{w}$ and $\tau^{w}$ refer to the axial $w$-modes, and $\nu^{B H}$ and $\tau^{B H}$ to the black hole

\begin{tabular}{lllllll}
\hline$\frac{M}{R}$ & $\nu^{s}$ & $\tau^{s}$ & $\nu^{w}$ & $\tau^{w}$ & $\nu^{B H}$ & $\tau^{B H}$ \\
\hline 0.4167 & 8.6293 & $1.52 \cdot 10^{-3}$ & 11.1738 & $1.70 \cdot 10^{-4}$ & 8.9300 & $7.49 \cdot 10^{-5}$ \\
& - & - & 14.2757 & $8.03 \cdot 10^{-5}$ & 8.2848 & $2.43 \cdot 10^{-5}$ \\
& & & & & & \\
& - & - & 18.2232 & $5.70 \cdot 10^{-5}$ & 7.1952 & $1.39 \cdot 10^{-5}$ \\
& - & - & 22.6669 & $4.88 \cdot 10^{-5}$ & 6.0099 & $0.95 \cdot 10^{-5}$ \\
\hline 0.4386 & 4.4333 & 10.8 & 10.4128 & $5.45 \cdot 10^{-4}$ & & \\
& 6.0168 & $2.50 \cdot 10^{-1}$ & 11.9074 & $2.91 \cdot 10^{-4}$ & & \\
& 7.5462 & $1.44 \cdot 10^{-2}$ & 13.4813 & $2.07 \cdot 10^{-4}$ & & \\
& 8.9891 & $1.83 \cdot 10^{-3}$ & 15.1428 & $1.67 \cdot 10^{-4}$ & & \\
\hline 0.4425 & 2.6041 & $5.38 \cdot 10^{3}$ & 10.7852 & $7.60 \cdot 10^{-4}$ & & \\
& 3.5427 & $1.69 \cdot 10^{2}$ & 11.6922 & $5.34 \cdot 10^{-4}$ & & \\
& 4.4802 & $1.22 \cdot 10^{1}$ & 12.6138 & $4.22 \cdot 10^{-4}$ & & \\
& 5.4127 & $1.37 \cdot 10^{-1}$ & 13.5512 & $3.56 \cdot 10^{-4}$ & & \\
\hline & & & & & & \\
\hline
\end{tabular}

signal emitted in this process contains both the frequency of the $w$-modes of the collapsing star, and those of the quasi-normal modes of the newly born black hole 38 .

The polar modes are classified following a scheme, introduced by Cowling in Newtonian gravity in 1942 65], based on the restoring force which prevails when the generic fluid element is displaced from the equilibrium position. They are said $g$-modes, or gravity modes, if the restoring force is due to buoyancy, and $p$-modes if it is due to pressure gradients. The mode frequencies are ordered as follows

$$
. . \omega_{g_{n}}<. .<\omega_{g_{1}}<\omega_{f}<\omega_{p_{1}}<. .<\omega_{p_{n}} .
$$

and are separated by the frequency of the fundamental mode ( $f$-mode), which has an intermediate character between $g$ - and $p$ - modes. In addition, general relativity predicts the existence of polar $w$-modes, that are very weakly coupled to fluid motion and are similar to the axial $w$-modes [66]. Their frequencies are typically higher than those of the fluid modes $(g, f$ and $p)$.

The relevance of the different modes to gravitational wave emission depends on several factors, first of all on the amount of energy which can be stored into a given mode. Moreover, it depends on the presence of other dissipative processes that may compete with GW-emission in removing energy from the star; for instance, neutrino diffusion or viscosity, if the oscillating 
star is a newly born, hot neutron star. Last, but not least, of crucial importance are the values of the mode frequencies: for instance the $w$-modes of neutron stars have typical frequencies of the order of $10 \mathrm{kHz}$ or higher, far too high to be detectable by ground based interferometers in their present or advanced configuration.

Numerical simulations of the most energetic astrophysical processes, like core collapse to a neutron star (NS) or binary coalescence leading to NS formation, indicate that the mode which is most excited is the fundamental mode. For this reason we will now focus on this mode, discussing the information the $f$-mode frequency, $\nu_{f}$, carries on the inner structure of neutron stars.

It is worth mentioning that typical values of $f$-mode damping times are of the order of a few tenths of seconds; consequently, the excitation of the $f$-mode would appear, in the Fourier transform of the gravitational signal, as a sharp peak. Therefore, the $f$-mode frequency could, in principle, be extracted from the detector noise by an appropriate data analysis (provided $\nu_{f}$ falls in the detection bandwidth of some GW-detector).

From Newtonian gravity we know that the $\nu_{f}$ scales with the average density of the star $\nu_{f} \sim\left(\frac{M}{R^{3}}\right)^{1 / 2}$; as shown in [67,68, where $\nu_{f}$ has been computed for a variety of equations of state proposed to describe matter in a NS, a similar scaling law holds also in general relativity.

At densities exceeding the equilibrium density of nuclear matter, $\rho_{0}=$ $2.67 \times 10^{14} \mathrm{~g} / \mathrm{cm}^{3}$, the fluid in the inner core of a NS is basically a gas of interacting nucleons. The equations of state proposed in the literature to describe this kind of matter are derived within two main, different approaches: nonrelativistic nuclear many-body theory (NMBT) and relativistic mean field theory (RMFT); we will now show how different ways of modeling hadronic interactions affect the pulsation properties of the star. In what follows we shall summarize the main results of a study we have done in 68, where we have selected a restricted number of EOS obtained within the NMBT and the RMFT approach. To describe the inner and outer crust of the NS, we have used the Baym-Pethick-Sutherland EOS [69] and the Pethick-RavenhallLorenz EOS [70], respectively.

The EOS we choose to describe NS matter at $\rho>\rho_{0}$, are the following. For the NMBT approach we select two groups of EOS: Group I, named (APR1 , APR2 , APRB200 , APRB120 ), and Group II, named (BBS1 , BBS2 ), respectively. In both cases matter is composed of neutrons, protons, electrons and muons in weak equilibrium, and the dynamics is described by a non-relativistic Hamiltonian which includes phenomenological potentials that describe two- and three-nucleon interactions. The potential are obtained from fits of existing scattering data. For all EOS the two-body potential is $v_{18}$, whereas the three-body potential is Urbana IX for Group I, and Urbana VII for Group II.

A first major difference between the two groups is that in Group I the ground state energy is calculated using variational techniques [71,72], whereas in Group II is calculated using G-matrix perturbation theory [73]. There are also differences among the EOS in each group: 


\section{- Group I}

APR2 is an improved version of the APR1 model. In APR1 nucleonnucleon potentials describe interactions between nucleons in their center of mass frame, in which the total momentum $\mathbf{P}$ vanishes. In the APR2 the two-nucleon potential is modified including relativistic corrections which arise from the boost to a frame in which $\mathbf{P} \neq 0$, up to order $\mathbf{P}^{2} / m^{2}$. These corrections are necessary to use the nucleon-nucleon potential in a locally inertial frame associated to the star. As a consequence of this change, the three-body potential also needs to be modified in a consistent fashion.

The EOS APRB200 and APRB120 are the same as APR2 up to $\sim 4 \rho_{0}$, but at higher density there is a phase of deconfined quark matter described within the MIT bag model. The mass of the strange quark is assumed to be $m_{s}=150 \mathrm{MeV}$, the coupling constant describing quarks interaction is set to $\alpha_{s}=0.5$, and the value of the bag constant is 200 $\mathrm{MeV} / \mathrm{fm}^{3}$ for APRB200 and $120 \mathrm{MeV} / \mathrm{fm}^{3}$ for APRB120. We will discuss in some more detail quark matter in the next section. The phase transition from nuclear matter to quark matter is described requiring the fulfillment of Gibbs conditions, leading to the formation of a mixed phase, and neglecting surface and Coulomb effects [71,74. Thus, these stars are hybrid stars.

- Group II

The main difference between the equations of state BBS1 and BBS2 is that in BBS2 strange heavy baryons $\left(\Sigma^{-}\right.$and $\left.\Lambda^{0}\right)$ are allowed to form in the core. Neither BBS1 nor BBS2 include relativistic corrections.

As representative of the RMFT, we choose the EOS named G240 . Matter composition includes leptons and the complete octet of baryons (nucleons, $\Sigma^{0, \pm}, \Lambda^{0}$ and $\Xi^{ \pm}$). Hadron dynamics is described in terms of exchange of one scalar and two vector mesons. It should be reminded that in this case, the EOS is obtained within the mean field approximation [75].

For any of the above EOS we have solved the TOV equations for different values of the central density, finding the equilibrium configurations. Then, for each EOS and for each equilibrium model, we have solved the equations of stellar perturbations finding the $f$-mode frequency, $\nu_{f}$. The results are shown in Fig. 3 where we plot $\nu_{f}$ as a function of the mass, up to the maximum mass allowed by each EOS. From this picture we learn the following. Comparing the values of $\nu_{f}$ for APR1 and APR2 we immediately see that the relativistic corrections and the associated redefinition of the three-body potential, which improve the Hamiltonian of APR2 with respect to APR1, play a relevant role, leading to a systematic difference of about $150 \mathrm{~Hz}$ in the mode frequency. Conversely, the presence of quark matter in the star inner core (EOS APRB200 and APRB120 ) does not seem to significantly affect the pulsation properties of the star. We also see that the frequencies corresponding to the BBS1 and APR1 models, which are very close at $M \lesssim 1.4 M_{\odot}$, diverge for larger masses. This behavior can be traced back to the different treatments of three-nucleon interactions, whose role in shaping the EOS becomes more and more important as the star mass (and central density) increases: while the variational approach of ref. [71] used to derive the EOS APR1 naturally 


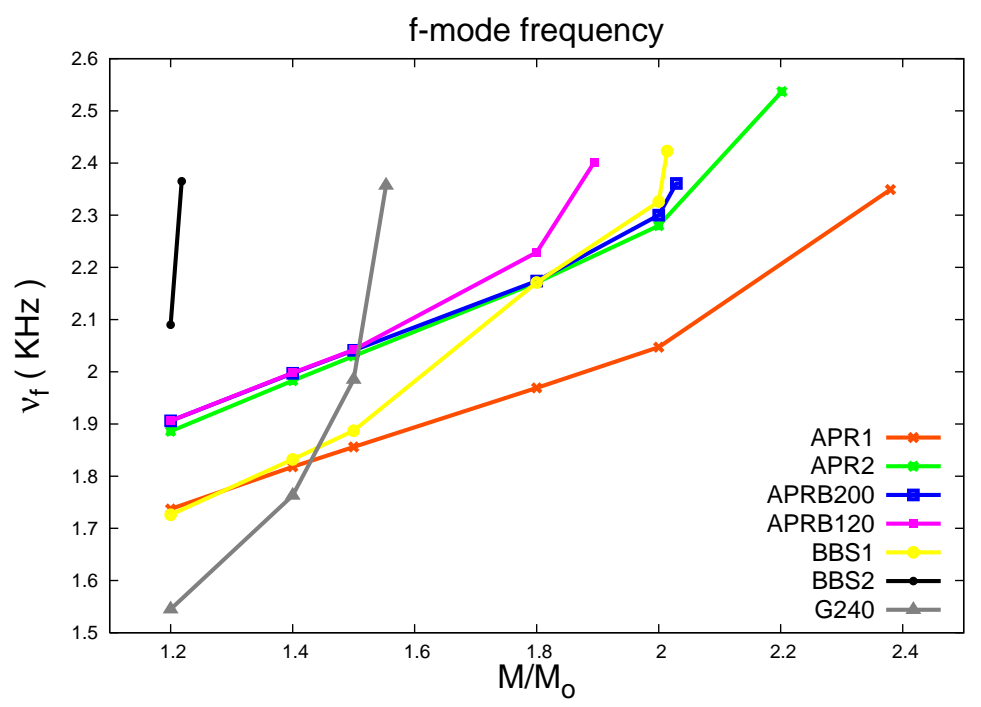

Fig. 3 The frequency of the fundamental mode is plotted as a function of the mass of the star for the selected EOS (see text).

allows for inclusion of the three-nucleon potential appearing in the Hamiltonian, in G-matrix perturbation theory used to derive the EOS BBS1 the three-body potential has to be replaced with an effective two-nucleon potential, obtained by averaging over the position of the third particle [76.

The transition to hyperonic matter, predicted by the BBS2 model, produces a considerable softening of the EOS, and leads to stable NS configurations of very low mass $\left(=1.218 M_{\odot}\right)$. As a consequence of the softening of the EOS, the average density increases and so does the $f$-mode frequency, which is significantly higher than that obtained for other EOS for the same mass.

It is also interesting to compare the $f$-mode frequencies corresponding to models BBS2 (derived within the RMFT approach) and G240 (derived using the NMBT approach), as they both predict the occurrence of heavy strange baryons, but are obtained from different theoretical approaches. The behavior of $\nu_{f}$ shown in Fig. 3 directly reflects the relations between mass and central density; indeed, for a given mass, larger central density correspond to smaller radii, and therefore to larger average density. Consequently, we can say that higher frequencies correspond to larger central densities $\rho_{c}$. For example, the NS configurations of mass $1.2 M_{\odot}$ correspond to $\rho_{c} \sim 7 \cdot 10^{14}$ $\mathrm{g} / \mathrm{cm}^{3}$ for G240, and to a larger central density, $\rho_{c} \sim 2 \cdot 10^{15} \mathrm{~g} / \mathrm{cm}^{3}$ for BBS2 . On the other hand, the G240 model requires a central density of $\sim$ $2.5 \cdot 10^{15} \mathrm{~g} / \mathrm{cm}^{3}$ to reach a mass of $\sim 1.55 M_{\odot}$ and a value of $\nu_{f}$ equal to that of the BBS2 model.

From the above discussion we see that the frequency of the fundamental mode carries interesting information on the different ways of modeling hadronic interactions. 
3.3 Can a star be made of quarks?

Another interesting possibility is that a compact star is a strange star, namely one that, except for a thin outer crust, is entirely made of a degenerate gas of up, down and strange quarks. That such stars may exist, was suggested by Witten many years ago [7]; since then, from time to time the observation of a very compact (or what seems to be a very compact) object revives the question whether strange stars can actually exist. Thus, it is interesting to see whether gravitational waves would be able to provide an answer to this question. For this reason in 78 we computed the $f$-mode eigenfrequency of strange stars, modeled using the MIT bag model [79. It may be noted that, due to the complexity of the fundamental theory of strong interactions between quarks (Quantum Chromo-Dynamics, or QCD), theoretical studies of strange stars are necessarily based on models, and the MIT bag model is one of the most used in the literature. According to such model, quarks occur in color neutral clusters confined to a finite region of space - the bag - the volume of which is limited by the pressure of the QCD vacuum (the bag constant $B$ ); in addition, the residual interactions between quarks are assumed to be weak, and therefore are treated in low order perturbation theory in the color coupling constant $\alpha_{s}$. Thus, the parameters of the model are the masses of up, down and strange quarks, $\alpha_{s}$ and the bag constant $B$.

From the Particle Data Book we learn that the mass of the up and down quarks are of the order of few $\mathrm{MeV}$, negligible with respect to that of the strange quark, the value of which is in the ranges $(80-155) \mathrm{MeV}$. The value of the coupling constant $\alpha_{s}$ is constrained by the results of hadron collision experiments to range within $(0.4-0.6)$.

In early applications of the MIT bag model $B, \alpha_{s}$ and $m_{s}$ were adjusted to fit the measured properties of light hadrons (spectra, magnetic moments and charge radii). According to these studies $B$ was shown to range from $57.5 \mathrm{MeV} / \mathrm{fm}^{3}$ 80 to $351.7 \mathrm{MeV} / \mathrm{fm}^{3}$ 81; however, the requirement that strange quark matter be absolutely stable at zero temperature and pressure implies that $B$ cannot exceed the maximum value $B_{\max } \approx 95 \mathrm{MeV} / \mathrm{fm}^{3}[82$. For values of $B$ exceeding $B_{\max }$, a star entirely made of deconfined quarks is not stable, and quark matter can only occupy a fraction of the available volume as in the models APRB200 and APRB120 considered above. Thus, if we want to study bare strange stars we need to restrict the values of $B$ in the range $\in(57-95) \mathrm{MeV} / \mathrm{fm}^{3}$. In our analysis we have systematically explored the following range of parameters

$$
m_{s} \in(80-155) \mathrm{MeV}, \quad \alpha_{s} \in(0.4-0.6), \quad B \in(57-95) \mathrm{MeV} / \mathrm{fm}^{3},
$$

computing the corresponding stellar configurations up to the maximum mass, and the corresponding $f$-mode frequencies. The results are summarized in figure 4. There we plot $\nu_{f}$ as a function of the mass of the star, both for strange stars and for the neutron/hybrid stars described in the previous section. The shaded region covers the range of parameters of the MIT bag model (22).

From figure 4 we can extract the following information. First of all strange stars cannot emit gravitational waves with $\nu_{f} \lesssim 1.7 \mathrm{kHz}$, for any value of the 


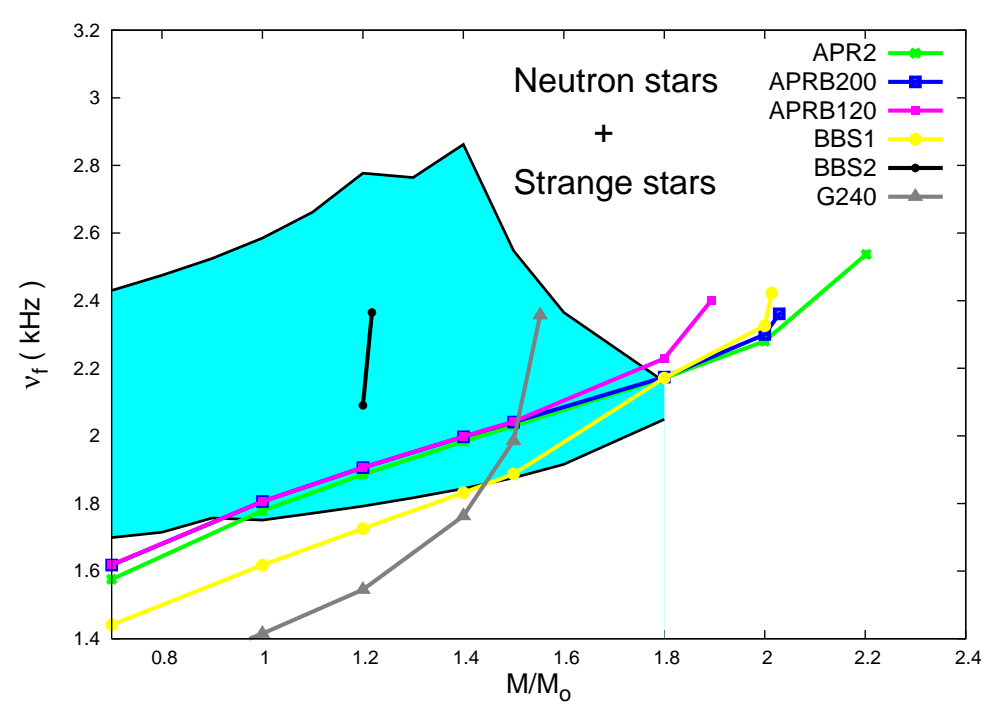

Fig. 4 The frequency of the fundamental mode is plotted as a function of the mass of the star, for neutron/hybrid stars (continuous lines) and for strange stars modeled using the MIT bag model, spanning the set of parameters indicated in (22) (dashed region).

mass in the range we consider. Note that $1.8 M_{\odot}$ is the maximum mass above which no stable strange star can exist. There is a small range of frequency where neutron/hybrid stars are indistinguishable from strange stars; however, there is a large frequency region where only strange stars can emit. For instance if $M=1.4 M_{\odot}$, a signal with $\nu_{f} \gtrsim 2 \mathrm{kHz}$ would belong to a strange star. Even if we do not know the mass of the star (as it is often the case for isolated pulsars) the knowledge of $\nu_{f}$ allows to gain information about the source nature; indeed, if $\nu_{f} \gtrsim 2.2 \mathrm{kHz}$, apart from a very narrow region of masses where stars with hyperons would emit (EOS BBS2 and G240), we can reasonably exclude that the signal is emitted by a neutron star.

In addition, it is possible to show that if a signal emitted by an oscillating strange star would be detected, since $\nu_{f}$ is an increasing function of the bag constant $B$ it would be possible to set constraints on $B$ much more stringent than those provided by the available experimental data [8].

\section{Are we ready for gravitational wave asteroseismology?}

In this section we want to discuss whether we are in a position to establish what is (or are) the equation(s) of state appropriate to describe matter at supranuclear densities, using gravitational wave signals. This is of course a fundamental question, because the energies prevailing in the inner core of neutron stars are unaccessible to high energy experiments on Earth.

The answer is, unfortunately, negative, essentially for two reasons. The first is that ground based interferometers do not have, at present, sufficiently 
high sensitivity at frequencies in the range $\sim 1.5-3 \mathrm{kHz}$, typical for $\nu_{f}$. Feasibility studies of interferometric, high frequency detectors have been considered in recent years 83 , and high frequency, wide-band, resonant detectors are under study [84]; however, if we restrict to Virgo or LIGO in their present configuration, to detect a signal emitted by a NS pulsating in the $f$-mode in our Galaxy, with a signal-to-noise ratio of 5 , the energy stored into the mode should be $E_{f-\text { mode }} \sim 6 \cdot 10^{-7} M_{\odot} c^{2}$. In order to understand whether it is plausible that the fundamental mode is excited to such an extent, we can refer either to the results of numerical simulations, or to astrophysical data. Numerical simulations of gravitational collapse show that the amount of energy released in gravitational waves is in the range $E_{G W}$ tot $\sim\left[10^{-9}-10^{-6}\right] M_{\odot} c^{2}$ 85. Although computed waveforms show that the $f$-mode is excited, at present there is no conclusive indication on the fraction of $E_{G W}$ tot which may go into that mode, since it depends on the initial conditions and on the physical assumptions that are made in modeling the collapse. Just to mention one, usually numerical simulations assume axisymmetric collapse, but in the non axisymmetric case energy released in GWs may be higher.

Thus, we can only say that $E_{f-\text { mode }} \sim 6 \cdot 10^{-7} M_{\odot} c^{2}$ is not totally unreasonable. Unfortunately, gravitational collapse is a rare event (about 3 events every hundred years, per galaxy), and if we restrict to our Galaxy chances to detect one in our lifetime are not too high.

The $f$-mode may also be excited in a cold, old neutron star as a consequence of a glitch. Glitches are sudden changes in the rotation frequency of the neutron star crust. They are observed in many pulsars and are thought to be related to quakes occurring in the solid structures such as the crust, the superfluid vortices and, perhaps, the lattice of quark matter in the stellar core $[86,87,88$. The rotational energy released in a glitch is $\Delta E \approx I \Omega \Delta \Omega$, where $I$ is the moment of inertia of the star, and typical spin variations are $\Delta \Omega / \Omega \approx 10^{-6}-10^{-8}$. For the glitches observed in the Crab and Vela pulsars observations give $\Delta E \simeq 2 \cdot 10^{-13} M_{\odot} c^{2}$ and $\Delta E \simeq 3 \cdot 10^{-12} M_{\odot} c^{2}$, respectively. As before, we do not know which fraction of $\Delta E$ goes in the $f$-mode excitation; in any event being $\Delta E$ so small, we can conclude that there is no hope to detect anything like this with the detectors that are actually in operation.

The second reason why we are far from being able to infer the EOS of matter in the inner core of a NS using gravitational waves, is that the EOS proposed in the literature only loosely constrain the dynamics of nuclear matter. This statement can be made more clear by the following example. Since from Newtonian gravity we know that $\nu_{f}$ scales as the square root of the average density, we expect a similar relation to hold also in general relativity. Indeed, a linear relation between $\nu_{f}$ and $\sqrt{\frac{M}{R^{3}}}$ has been obtained in [67, fitting the data referring to stars modeled with a large set of EOS. In 68. a similar fit has been found using the same EOS considered in previous sections; since the two fits are similar, in what follows we shall explicitly use our fit:

$$
\nu_{f}=a+b \sqrt{\frac{M}{R^{3}}}, \quad a=0.79 \pm 0.09, \quad b=33 \pm 2
$$


where $a$ is in $\mathrm{kHz}$ and $b$ in $\mathrm{km} \cdot \mathrm{kHz}$. The fit is plotted in figure 5 versus $\sqrt{\frac{M}{R^{3}}}$, together with the $f$-mode frequencies corresponding to the stellar models considered in figure 3. Similarly, the first $p$-mode frequency can be fitted as

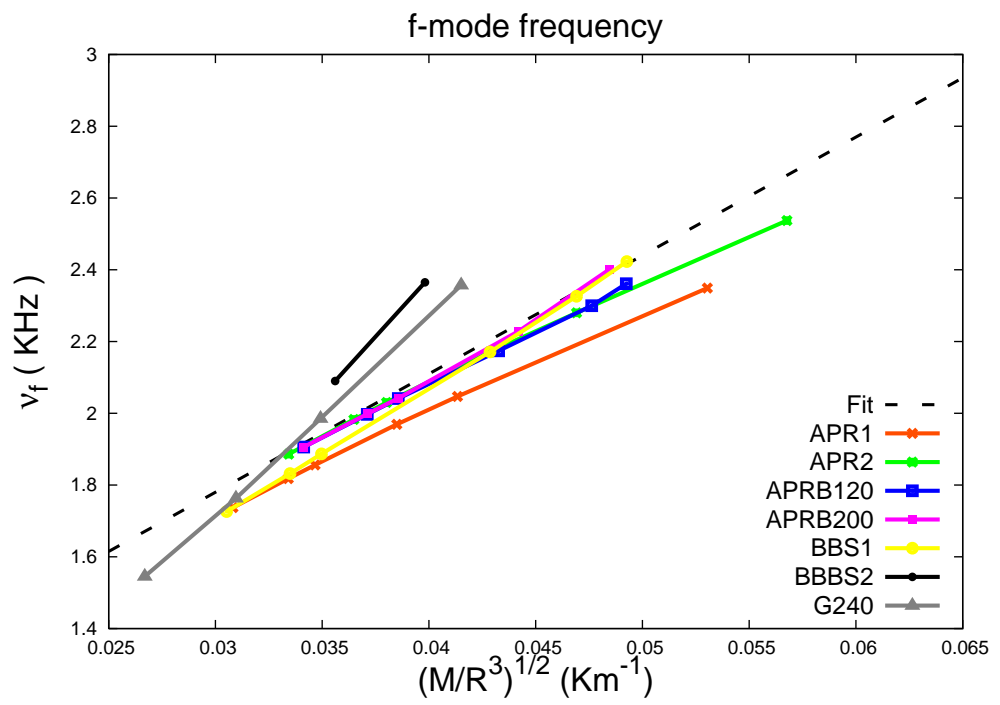

Fig. 5 The frequency of the fundamental mode is plotted as a function of the square root of the average density for the EOS considered in this paper. We also plot the fit given in eq. (23)

a function of the stellar compactness $M / R$ as follows

$$
\nu_{p_{1}}=\frac{1}{M}\left[a+b \frac{M}{R}\right], \quad a=-1.5 \pm 0.8, \quad b=79 \pm 4,
$$

where $a$ and $b$ are in $\mathrm{km} \cdot \mathrm{kHz}$. In these fits, frequencies are expressed in $\mathrm{kHz}$, masses and radii in $\mathrm{km}$.

Let us now consider a star belonging to the EOS APR2, with mass $M=1.4 M_{\odot}$ and radius $R=11.58 \mathrm{~km}$. Let us assume that, as a consequence of some astrophysical process, both the fundamental mode and the first $p$ mode are excited and that the emitted gravitational wave has been detected. With the detected values of $\nu_{f}$ and $\nu_{p_{1}}$ (which we know to be $\nu_{f}=1.983 \mathrm{kHz}$, $\nu_{p}=6.164 \mathrm{kHz}$ ) we could plot the fits (23) and (24) in the $(R, M)$-plane, and we would find what is shown in figure 6 the two curves intersect in a point which corresponds to $M=1.30 M_{\odot}, R=11.36 \mathrm{~km}$; consequently, we would be able to estimate the values of the mass and of the radius with an error of $7 \%$ and $2 \%$, respectively. This would be great, but unfortunately, the fit comes with error bars. If, for instance we plot the curve referring to the $f$-mode, and we show the entire region where the parameters $a$ and $b$ can vary (the dashed region in figure 7), we see that the error bar induces a 


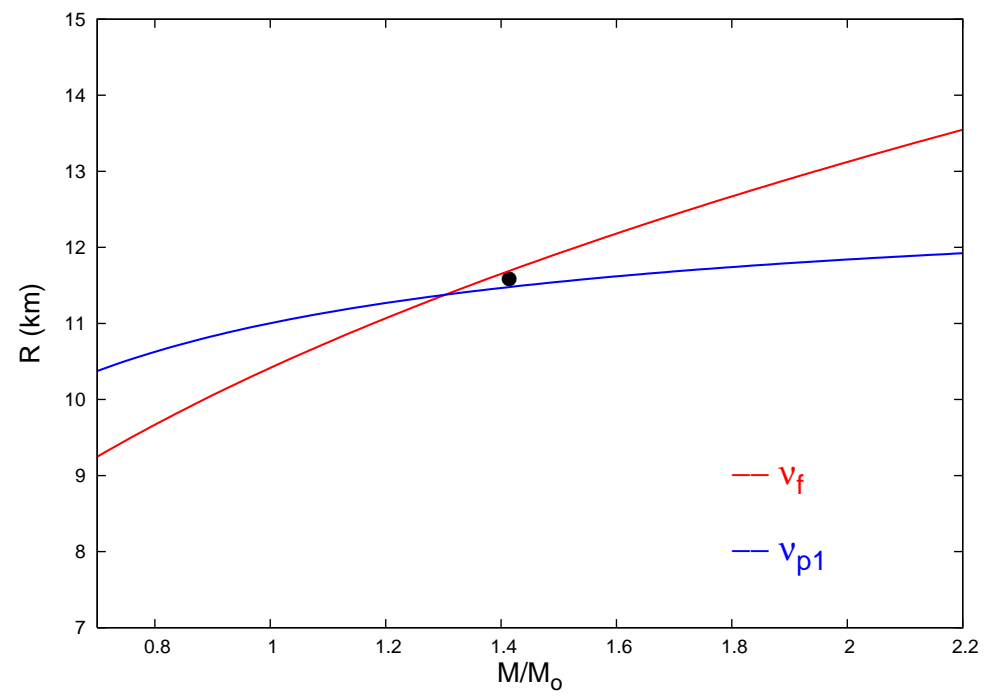

Fig. 6 The fits (23) and (24) are plotted in the $R-M$-plane, assuming the mode frequencies $\nu_{f}=1.983 \mathrm{kHz}$ and $\nu_{p}=6.164 \mathrm{kHz}$ have been identified in a detected signal. The black dot corresponds to the true values of the mass and radius of the emitting star.

very large error on $R$; so large indeed that, even knowing the mass, we would estimate $R$ with an error of the order of $18 \%$.

Therefore, for the time being, we can only say that gravitational wave asteroseismology will become possible when GW-detectors will become more sensitive to the high frequency region, and when nuclear matter studies will put tighter constraints on the parameters that characterize the equation of state of superdense matter.

Since science always looks forward for expanding the horizon of knowledge, we are confident that one day this will be possible.

\section{References}

1. www.ligo.caltech.edu; www.virgo.infn.it

2. Kokkotas K.D. and Schmidt B.G. 1999 Living Rev. Rel. 2, 2

3. Nollert H.P. 1999 Class. Quant. Grav. 16 R159

4. Shapley H. 1914 Astrophys. J. 40448

5. Eddington A.S. 1918 Mon Not Roy. Astron. Soc. 79, 2

6. Regge T. and Wheeler J.A. 1957 Phys. Rev. 108, 1063

7. Zerilli J.F. 1970 Phys. Rev. D 2, 2141; Zerilli J.F. 1970 Phys. Rev. Lett. 24, 737

8. Misner C.W., Thorne K S and Wheeler J A 1973, Gravitation, W.H. Freeman $\&$ C, New York

9. Vishveshwara C.V. 1970 Phys. Rev. D 1, 2870

10. Press W.H. 1971 Astrophys. J. 170, L105

11. Chandrasekhar S. and Detweiler S.L. 1975 Proc. R. Soc. Lond. A 344, 441

12. Chandrasekhar S. 1984 The mathematical theory of black hole, Claredon Press, Oxford 


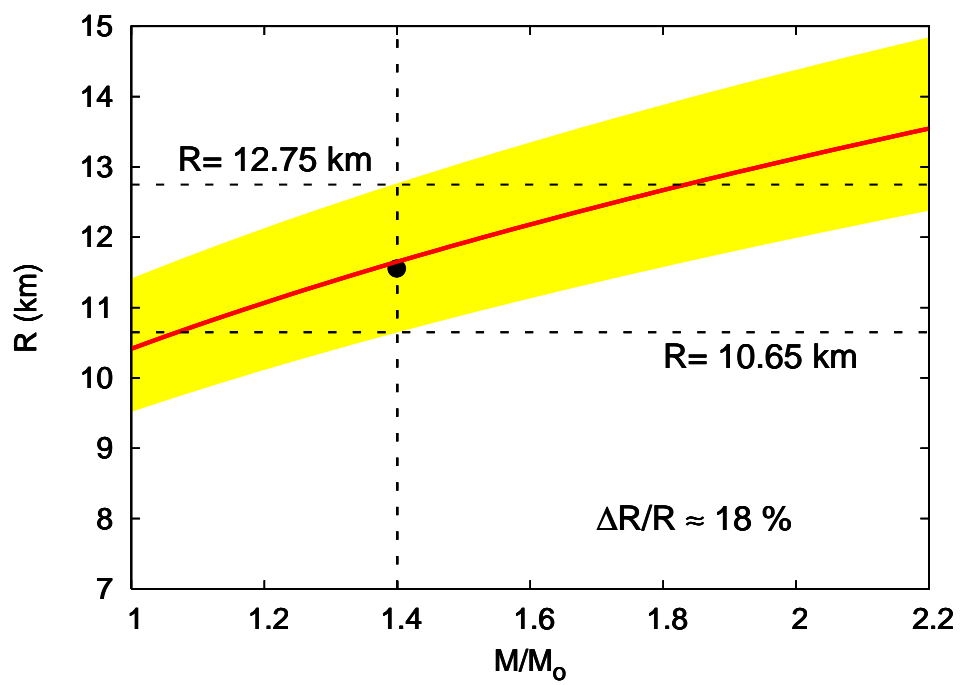

Fig. 7 The fit (23) is plotted in the $R-M$-plane including the error bars on the fit parameters (dashed region).

13. Ghez A.M., Salim S., Hornstein S.D., Tanner A., Morris M., Becklin E.E. and Duchene G. 2005, Astrophys. J. 620, 744

14. Schutz B.F. and Will C.M. 1985 Astrophys. J. Lett. 291, L33; Iyer S. and Will C.M. 1987 Phys. Rev., D 35, 3621; Iyer S. 1987 Phys. Rev. D 35, 3632 (1987); Kokkotas K.D. and Schutz B.F. 1988 Phys. Rev. D 37, 12

15. Guinn J.W., Will C.M., Kojima Y. and Schutz B.F. 1990 Class. Quantum Grav. 7, L47

16. Leaver E.W. 1985 Proc. R. Soc. Lond. A 402, 285

17. Andersson N.,1992 Proc. R. Soc. Lond. A 439, 47

18. Nollert H.-P. 1993 Phys. Rev. D 47, 5253; Andersson N. 1993 Class. Quantum Grav. 10, L61; Barreto A.S. and Zworski M. 1997 Math. Res. Lett. 4, 103; Padmanabhan T. 2004 Class. Quantum Grav. 21, L1; Motl L. 2003 Adv. Theor. Math. Phys. 6, 1135

19. Fiziev P.P. 2006 Class. Quantum Grav. 23, 2447

20. Teukolsky S. 1972 Phys. Rev. Lett. 29, 1114; Teukolsky S. 1973 Astrophys. J. $\mathbf{1 8 5}, 635$

21. Starobinski A.A. and Churilov S.M. 1973 Soviet JEPT 38, 1

22. Press W.H. and Teukolsky S. 1973 Astrophys. J. 185, 649

23. Detweiler S.L. 1977 Proc. R. Soc. Lond. A 352, 381; Detweiler S.L. 1979 in Sources of Gravitational Radiation, edited by L. Smarr, Cambridge, England, 211; Detweiler S.L. 1978 Astrophys. J. 225, 687; Detweiler S.L. 1980 Astrophys. J. 239, 292

24. Seidel E., Iyer S. 1990 Phys. Rev D 41, 374

25. Kokkotas K.D. 1991 Class.Quantum Grav. 8, 2217

26. Onozawa H. 1997 Phys. Rev. D 55, 3593

27. Ferrari V. and Mashoon B. 1984 Phys. Rev. Lett. 52, 1361; Ferrari V. and Mashoon B. 1984 Phys. Rev. D 30, 295

28. Berti E., Cardoso V., Kokkotas K.D. and Onozawa H. 2003 Phys. Rev. D 68, 124018; Berti E., Cardoso V. and Yoshida S. 2004 Phys. Rev. D 69, 124018

29. Davis M., Ruffini R., Press W.H. and Price R.H. 1971 Phys. Rev. Lett 27, 1466

30. Davis M., Ruffini R. and Tiommo J. 1971 Phys. Rev. D 5, 2932; Ferrari V. and Ruffini R. 1981 Phys. Lett. B 98, 381 
31. Haugan M.P., Shapiro S.L. and Wasserman I. 1982 Astrophys. J. 257283 ; Shapiro S.L. and Wasserman I. 1982 Astrophys. J. 260 838; Pertich L.I., Shapiro S.L. and Wasserman I. 1985 Astrophys. J. Suppl. S 58297

32. Oohara K. and Nakamura T. 1983 Phys. Lett. A 98403

33. Berti E., Cardoso V. and Will C.M. 2006 AIP Conf. Proc. 848, 687

34. Nakamura T., Oohara K. and Kojima Y. 1987 Prog. Theor. Phys. Suppl. 90 218

35. Pretorius F. 2005 Phys. Rev. Lett 95 121101; Campanelli M., Lousto C.O., Marronetti P. and Zlochower Y., 2006 Phys. Rev. Lett 96 111101; Baker J.G., Centrella J., Choi D.-I., Koppitz M. and van Meter J. 2006 Phys. Rev. Lett 96 111102

36. Baker J.G., Campanelli M., Pretorius F. and Zlochower Y. 2007 Class. Quant. Grav. 24, S25

37. Campanelli M., Lousto C.O. and Zlochower Y. 2006 Phys. Rev. D 74, 041501

38. Baiotti L., Hawke I., Rezzolla L. and Schnetter E. 2005 Phys. Rev. Lett. 94, 131101

39. Zanotti O., Font J.A., Rezzolla L. and Montero P.J. 2005 Mon Not Roy. Astron. Soc. 356, 1371; Nagar A., Zanotti O., Font J.A. and Rezzolla L. 2007 Phys. Rev. D 75, 044016

40. Ferrari V., Gualtieri L. and Rezzolla L. 2006 Phys. Rev. D 73, 124028

41. Leaver H.W. 1986 Phys. Rev. D 34, 384

42. Sun Y., and Price R. H. 1988 Phys. Rev. D 38, 1040; Andersson, N. 1995 Phys. Rev. D 51, 353; Nollert P. and Price R.H. 1999 J. Math. Phys. 40, 980; Glampedakis K. and Andersson N. 2001 Phys. Rev. D 64, 104021; Berti E. and Cardoso V. 2006 Phys. Rev. D 74, 104020

43. Price R.H. 1972 Phys. Rev. D 52419

44. Vishveshwara C.V. 1970 Phys. Rev. D 1, 2870

45. Wald R.M. 1979 J. Math. Phys. 20, 1056; Kay B.S. and Wald R.M. 1987 Class. Quantum Grav. 4, 893

46. Whiting B.F. 1989 J. Math. Phys. 30, 1301; Beyer H.R. 2001 Comm. Math. Phys. 221, 659

47. Horowitz G.T. and Hubeny V.E. 2000 Phys. Rev. D 62, 024027

48. Wang B., Lin C.Y. and Abdalla E. 2000 Phys. Lett. B 481, 79; Wang B., Molina C. and Abdalla E. 2001 Phys. Rev. D 63, 084001; Cardoso V. and Lemos J.P.S. 2001 Phys. Rev. D 63, 124015; Cardoso V. and Lemos J.P.S. 2001 -IT Phys. Rev. D 64, 084017; Berti E. and Kokkotas K.D. 2003 Phys. Rev. D 67, 064020; Cardoso V., Konoplya R. and Lemos J.P.S. 2003 Phys. Rev. D 68, 044024

49. Chan J.S.F. and Mann R.B. 1996 Phys. Rev. D 55, 7546; 1999 Phys. Rev. D 59, 064025.

50. Dreyer O. 2003 Phys. Rev. Lett. 90, 081301; Motl L. 2003 Adv. Theor. Math. Phys. 6, 1135

51. Motl L. and Neitzke A. 2003 Adv. Theor. Math. Phys. 7, 307; Cardoso V., Natario J. and Schiappa R. 2004 J. Math. Phys. 45, 4698; Natario J. and Schiappa R. 2004 Adv. Theor. Math. Phys. 8, 1001.

52. Cardoso V. and Lemos J.P.S. 2003 Phys. Rev. D 67, 084020; Konoplya R.A. 2003 Phys. Rev. D 68, 024018; Cardoso V., Lemos J.P.S. and Yoshida S. 2004 Phys. Rev. D 69, 044004

53. Stergioulas N. 2003 Living Rev. Rel. 6, 3

54. Thorne K.S. and Campolattaro A. 1968 Astrophys. J. 149, 591; 1970 Astrophys. J. 159, 847

55. Chandrasekhar S. and Ferrari V. 1991 Proc. R. Soc. Lond. A 432, 247;

56. Ferrari V. 1992 Phil. Trans. R. Soc. Lond. A 340, 423

57. Detweiler S.L. 1975 Astrophys. J. 201, 440

58. Lindblom L. and Detweiler S.L. 1983 Astrophys. J. Suppl. 53, 73

59. Lindblom L. and Splinter R.S. 1989 Astrophys. J. 345, 925

60. Detweiler S.L. and Lindblom L. 1985 Astrophys. J. 292, 12

61. Ipser J.R. and Price R.H. 1992 Phys. Rev. D 431768

62. Kokkotas K.D. 1994 Mon. Not. R. Ast. Soc. 2681015

63. Chandrasekhar S. and Ferrari V. 1991 Proc. R. Soc. Lond. 434, 449

64. Benhar O., Berti E. and Ferrari V. 1999 Mon. Not. Roy. Astron. Soc. 310, 797 
65. Cowling T.G. 1942 Mon. Not. R. Ast. Soc. 101, 367

66. Kokkotas K.D. and Schutz B.F. 1992 Mon. Not. R. Ast. Soc. 255119

67. Andersson N. and Kokkotas K.D. 1998 Mon. Not. R. Ast. Soc. 2991059

68. Benhar O., Ferrari V. and Gualtieri L. 2004 Phys. Rev. D 70, 124015

69. Baym G., Pethick C.J. and Sutherland P. 1971 Astrophys. J. 170, 299

70. Pethick C.J., Ravenhall B.G. and Lorenz C.P. 1995 Nucl. Phys. A 584, 675

71. Akmal A. and Pandharipande V.R. 1997 Phys. Rev. C 56, 2261

72. Akmal A., Pandharipande V.R. and Ravenhall D.G. 1998 Phys. Rev. C 58, 1804

73. Baldo M., Burgio G.F. and Schulze H.J. 2000 Phys. Rev. C, 61, 055801

74. Rubino R., thesis, Università "La Sapienza", Roma (unpublished); Benhar O. and Rubino R., to be published.

75. Glendenning N.K. 2000 Compact Stars (Springer, New York)

76. Lejeune A., Grangé P., Martzoff P. and Cugnon J. 1986 Nucl. Phys. A 453, 189

77. Witten E. 1984 Phys. Rev. D 30, 272

78. Benhar O., Ferrari V., Gualtieri L. and Marassi S. 2007 Gen. Rel. Grav. 39, 1323

79. Chodos A., Jaffe R.L., Johnson K., Thorne C.B. and Weiskopf W.F. 1974 Phys. Rev. D 9,3471

80. De Grand T., Jaffe R.L., Johnsson K. and Kiskis J. 1975 Phys. Rev. D 12, 2060

81. Carlson C.E., Hansson T.H. and Peterson C. 1983 Phys. Rev. D 27, 1556

82. Farhi E. and Jaffe R.L. 1984 Phys. Rev. D 30, 2379

83. www.astro.cf.ac.uk/geo/euro

84. www.dual.lnl.infn.it

85. Dimmelmeier H., Font J.A. and Müller E. 2002 Astron. Astrophys. 393, 523; Müller E., Rampp M., Buras R., Janka H.-T. and Shoemaker D.H. 2004 Astrophys. J. 603, 221;

Ott C.D., Burrows A., Dessart L. and Livne E. 2006 Phys. Rev. Lett. 96, 201102.

86. Anderson P.W. and Itoh N. 1975, Nature 256, 25

87. Ruderman M. 1976, Nature 203, 213

88. Pines D. and Alpar M.A. 1985, Nature 316, 27 